UPR-704T

\title{
Spontaneous Expulsion of Giant Lipid Vesicles Induced by Laser Tweezers
}

\author{
J. David Moroz and Philip Nelson \\ Department of Physics and Astronomy, University of Pennsylvania \\ Philadelphia, PA 19104 USA \\ Roy Bar-Ziv and Elisha Moses \\ Department of Physics of Complex Systems, \\ Weizmann Institute of Science, Rehovot 76100, Israel
}

\begin{abstract}
Irradiation of a giant unilamellar lipid bilayer vesicle with a focused laser spot leads to a tense pressurized state which persists indefinitely after laser shutoff. If the vesicle contains another object it can then be gently and continuously expelled from the tense outer vesicle. Remarkably, the inner object can be almost as large as the parent vesicle; its volume is replaced during the exit process. We offer a qualitative theoretical model to explain these and related phenomena. The main hypothesis is that the laser trap pulls in lipid and ejects it in the form of submicron objects, whose osmotic activity then drives the expulsion.
\end{abstract}

$7 / 96$ 


\section{Introduction}

Nature has carefully designed lipid molecules to yield extraordinary material properties. In water, lipids spontaneously self-assemble to form bilayer membranes, loose fluid confederations of molecules which nevertheless offer tremendous resistance to mechanical disruption, topology change, and permeation [1]. For example, despite a thickness of only a few nanometers pure bilayer membranes withstand surface tensions of more than $1 \mathrm{dyn} / \mathrm{cm}$ before rupture [2]. Of course the inert character of membranes is crucial for their role in cells as tough, flexible partitions. Cells have specific machinery to induce fusion, pore formation, etc. only when required. Understanding this machinery, and finding new artificial mechanisms for controlled bilayer reorganization, are key tasks for cell biology. In this paper we describe a new technique for selectively disrupting membranes using laser tweezers.

The development of laser tweezer technology has opened the door to the direct manipulation of micron-scale objects with adjustable piconewton scale forces. Two of us have applied tweezers directly to bilayer membranes to generate a number of striking phenomena, including dynamic shape transformations and membrane unbinding [3] [4⿴囗十 . A theoretical understanding has begun to emerge in which a primary effect of the laser is to create sudden surface tension $\Sigma$ in the bilayer, in the regime $10^{-4}-10^{-3} \mathrm{dyn} / \mathrm{cm}$ and controlled by the applied laser power [5] 6].

Perhaps most striking of all, laser tweezers can set up conditions which persist long after the laser is shut off and which lead to the spontaneous expulsion of interior objects from a vesicle without otherwise damaging either the "parent" or the "daughter" object [7] (Fig. 1). In the light of our earlier remarks it is remarkable that this dramatic membrane reorganization should happen for tensions a thousand times smaller than what is normally required. We will describe the observed phenomena and sketch a proposed mechanism.

Other authors have reported that vesicle expulsion can also be induced by chemical means [8]. The phenomena we will describe are triggered solely by laser action. Related phenomena are also seen in vesicles tensed using the micropipette method or osmotic shock [9], but these events require far greater tensions than the ones reported here. Finally, our work extends initial observations described in [7] in several ways which proved crucial to obtaining the theoretical picture introduced here. 

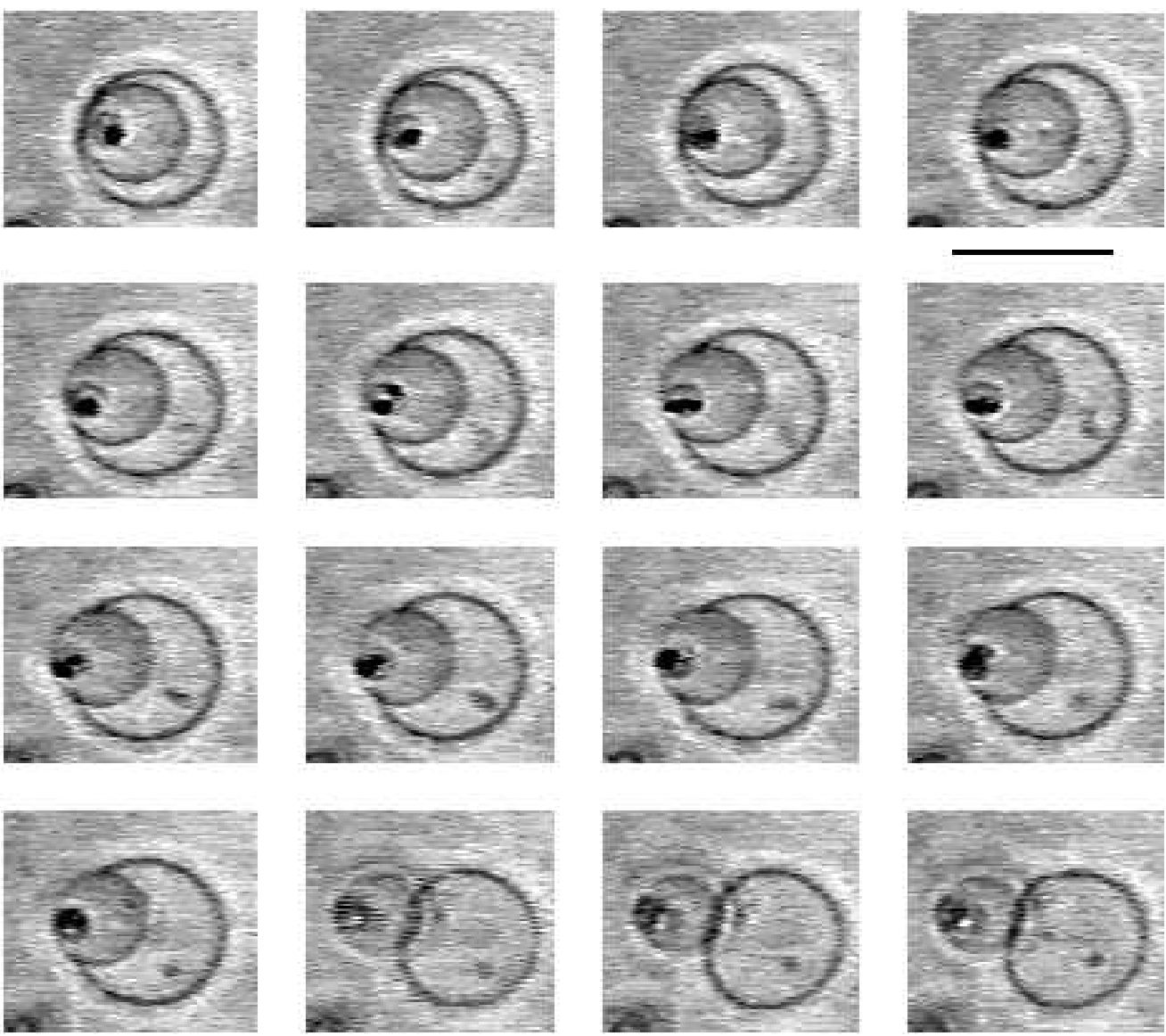

Fig. 1: Typical spontaneous giant expulsion event. Selected video frames are shown, separated by $0.12 \mathrm{~s}$. A large DMPC vesicle of radius $R=4.5 \mu \mathrm{m}$ in pure water (see text) initially contains a smaller daughter of radius $r=3.3 \mu \mathrm{m}$. The temperature was constant at $31^{\circ} \mathrm{C}$. The laser was focused to a spot about $0.3 \mu \mathrm{m}$ in diameter; an intensity of $6 \mathrm{~mW}$ was measured through this spot. After $22 \mathrm{~s}$ of tweezing the laser was shut off. Then the inner vesicle adhered to the outer one, waited $12 \mathrm{~s}$, and finally emerged as shown. In the final frames the daughter vesicle emerges rapidly, leaving the focal plane. The dark object trapped in the daughter vesicle plays no part in the expulsion process. Scale bar is $10 \mu \mathrm{m}$ in the horizontal direction; in the vertical direction the same bar is $10.6 \mu \mathrm{m}$, due to an asymmetry in our camera. 


\section{Methods}

We report results based on vesicles produced from dimyristoyl-phosphatidylcholine (DMPC, purchased from Sigma) using standard protocols [10. We have obtained similar results with stearoyl-oleoyl-phosphatidylcholine (SOPC) and digalactosyl-diglyeceride (DGDG) (Sigma), all of them uncharged, zwitterionic lipids. The experiments were performed in the fluid state of the membranes in a closed cell. In DMPC we varied the temperature from $26^{\circ} \mathrm{C}$ (just above the fluid transition) up to $85^{\circ} \mathrm{C}$ and observed expulsion in the full temperature range. We constrained the vesicles' initial volume osmotically. We used glucose concentrations ranging from $0 \mathrm{M}$ (we estimate that up to $1 \mathrm{mM}$ of uncontrolled impurities are always present in the solution) to $0.5 \mathrm{M}$, and consistently obtained expulsion. Even $1 \mathrm{mM}$ is sufficient to "clamp" the volume to high accuracy.

Our experimental apparatus uses an inverted optical microscope (Zeiss Axiovert 135TV with Planapochromat Ph3, 63x, NA 1.4 objective), in a commonly used optical tweezers setup [11]. A significant point lies in using the 488-514 nm Ar ion laser beam (Coherent, Innova 70) to produce the optical trap. This creates a tighter beam waist than infrared lasers and therefore a stronger electromagnetic field. The laser power ranged from 10 to $100 \mathrm{~mW}$. Heating at these power inputs is estimated to be lower than $\Delta T \approx 0.5 \mathrm{~K}[11]$.

Tension is produced in the membrane because the lipid has a higher refractive index than water and is pulled by the high electromagnetic field into the optical trap [5]. The electrostatic energy difference per unit area in the laser spot gives an estimate for the surface tension transmitted to the rest of the membrane, on the order of $\Sigma_{\text {laser }} \approx 10^{-3} \mathrm{dyn} / \mathrm{cm}$ for a power input of $25 \mathrm{~mW}$. For comparison, a flaccid vesicle of radius $R=10 \mu \mathrm{m}$ has $\Sigma$ in the range of $\kappa / R^{2} \approx 10^{-6} \mathrm{dyn} / \mathrm{cm}$, where $\kappa$ is the bending stiffness.

As the laser is applied to a flaccid vesicle, it gradually loses its fluctuations and becomes round and taut, a directly observable sign of tension in the membrane [7]. We have measured surface area losses on the order of $10 \%$ prior to expulsion, though other experiments show that much larger amounts can disappear. 


\section{Observations}

Fig. 1 shows a typical giant, spontaneous expulsion event.1 For concreteness we will focus our analysis on this event, but the same qualitative physics appears in many similar events. Fig. 2 defines notation. First the inner vesicle was drawn tense by tweezing for several seconds. The gradual increase in surface tension was clearly visible from the gradual suppression of visible thermal motion. In other experiments we have found that vesicles pressurized in this way remain tense indefinitely (at least hours), and that during tweezing the vesicle volume remained approximately fixed or grew slightly, while its area decreased gradually by about 10\%. Next the same procedure was repeated for the outer vesicle. Then the laser was shut off.

Often the tense inner vesicle wandered for some seconds in Brownian motion before encountering the outer wall. Upon close enough approach, adhesive forces snapped the vesicles together rapidly, sometimes visibly deforming the outer vesicle towards the inner before the two could draw together. Strong adhesive forces comparable to the applied membrane tension are expected on experimental and theoretical grounds [12].

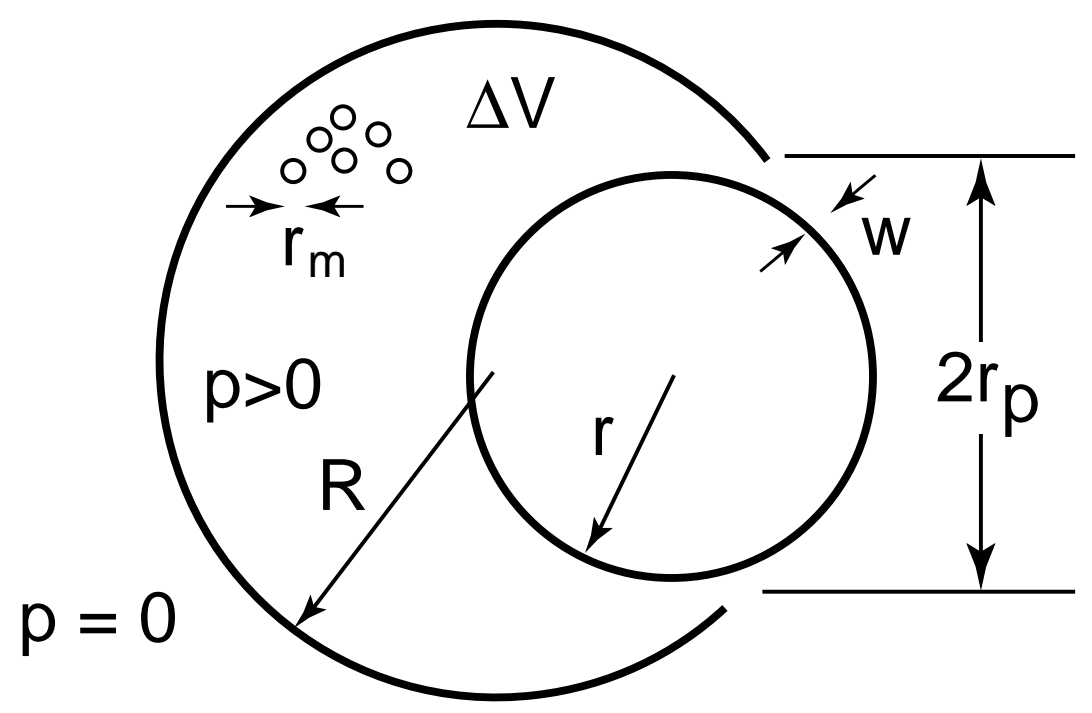

Fig. 2: Notation used in the text.

1 Small spontaneous expulsions, $r / R \leq 0.1$, were reported in [7]. The inflow described below was not visible in this regime. Also stimulated expulsions, where the laser was never turned off, have been seen for double-bilayer vesicles [7]. Here we study only spontaneous, giant expulsion. 
After a waiting period, typically 10 seconds, the inner vesicle began to emerge gradually. The point of initial tweezing was generally not related to the point of the subsequent exit. Though the daughter vesicle's volume was sometimes as large as $40 \%$ that of the parent, the parent always remained tense (i.e. spherical and nonfluctuating) through the halfway point. Most events slowed considerably near the halfway point, and some stalled and retracted prior to this. Every event which passed the halfway point completed rapidly, in one or two video frames.

In the final state the daughter was fully detached and could be readily pulled away by the usual tweezer manipulation. Throughout the process the volume and area of the daughter remained roughly constant (Fig. 3a), from which we infer that at least one of its monolayer walls was intact throughout. Remarkably, the area of the outer vesicle also remained constant through the halfway point (Fig. 3a). Thus not only was the final surface area equal to the original, but at every intermediate step the outer vesicle area (excluding the absent cap where the daughter is emerging) remained constant. Correspondingly the volume $\Delta V$ of the space between the vesicles was not constant but rather grew (Fig. $3 \mathrm{~b}$ ).
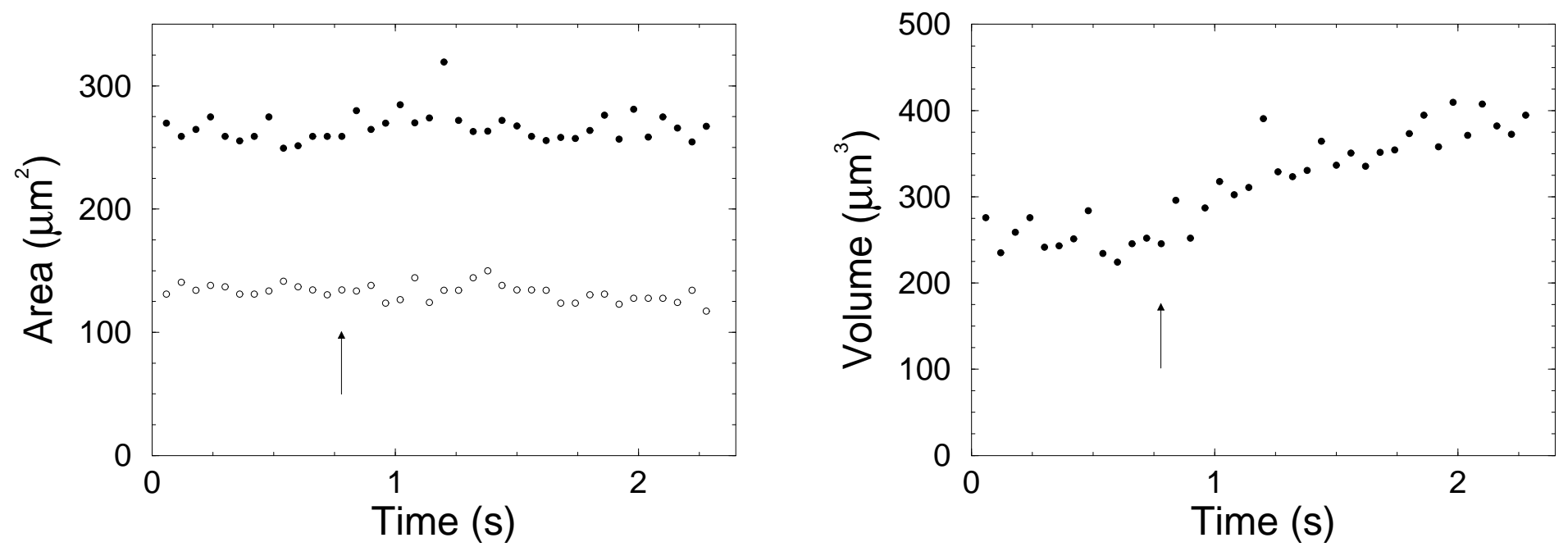

Fig. 3: (a) Surface area of inner and outer vesicles as a function of time. (b) Volume between inner and outer vesicles. The arrow refers to the first frame shown in Fig. 1; the points are spaced at intervals of $0.06 \mathrm{~s}$.

\section{Model}

One reason why spontaneous giant expulsion is so surprising is that after the laser is shut off, considerable energy must be stored somewhere in order to push open the exit 
pore, and yet there is no obvious elastic element in the system. The water is essentially incompressible, and the membrane itself can only stretch by a couple of percent, even when we account for entropic elasticity [2]. Where is the "spring" driving expulsion?

A second mystery is that whatever the thermodynamic force driving expulsion, it must overcome a nucleation energy barrier arising from the line energy $\gamma_{0}$ for creating pores. DMPC membranes withstand surface tensions of a few dyn/cm before rupture, implying that $\gamma_{0}$ is a few times $10^{-7}$ dyn (see [13]), and indeed vesicle expulsion without laser action (for example by micropipette aspiration [9]) requires tensions of this magnitude. The laserinduced tension is much less than this, and indeed tweezed vesicles without large included objects do remain tense indefinitely. What laser-induced mechanism creates an exit pore at low tension, but only in the presence of an interior object?

We get clues to both these puzzles when we note that the direct observation of membrane tension $\Sigma$ implies a corresponding hydrostatic pressure $p=2 \Sigma / R$ inside the large vesicle, and yet water is clearly seen to be entering, not leaving, the intermembrane space. This implies an osmotic flow mechanism: an excess of some solute in the interior maintains $p$ while pulling more water in to dilute the solute. Such a mechanism may at first sound paradoxical: with up to an atmosphere of osmotic pressure due to sugar on both sides of the outer vesicle, significant volume change would seem to require an absurdly large initial pressure difference. Indeed nothing of the sort can happen if pure water enters by permeation through the bilayer. (Moreover the known permeation rate of $70 \mu \mathrm{m} / \mathrm{sec}$ for DMPC [14] is much too small to give the observed influx.) If however a gap of width $w$ much larger than a sugar molecule opens (see Fig. 2), then sugar becomes osmotically irrelevant. Indeed as mentioned a wide range of sugar concentrations has little effect on expulsion. We will return later to the origin of the gap.

Thus we must identify some other osmotically-active solute driving expulsion. For this we focus on the area loss during the initial laser tweezing. Roughly $10 \%$ of the original $250 \mu \mathrm{m}^{2}$ of surface disappears permanently, leaving behind no visible scar or buildup. Our main physical hypothesis is that membrane area lost during the initial tweezing gets packaged into a suspension of small, optically unresolvable objects of size $r_{m}$, some of which get trapped inside the outer vesicle. This suspension is the "spring" we were looking for at the start of this section: its osmotic pressure is what stores the energy needed to open the exit pore.

These objects could be very small vesicles or membrane fragments. Perhaps more likely, the intense electric field in the laser spot may break apart the lipid molecules, 
creating a new chemical species analogous to the surfactant introduced directly by Menger and Gabrielson [8].2 Lipids are well known to be fragile; they degrade into lysolipids with heat or even the passage of time, with a significant reduction in their line energy [15]. The new species could then stabilize very small micelles, which would then avoid rejoining the membrane due to hydrodynamic interactions. Thus our hypothesis also explains the permanent area loss upon tweezing. Further work will be required to measure the size $r_{m}$. For concreteness we will illustrate our mechanism using the smallest reasonable value, $r_{m}=5 \mathrm{~nm}$. Once formed, the micelles can easily escape the laser trap. Their trapping energy is proportional to their area, $E \approx \Sigma_{\text {laser }} \cdot 4 \pi r_{m}{ }^{2}$, but their thermal energy $k_{B} T$ is much larger than this, so they readily diffuse away, making room for more material to enter the trap.

Let us estimate the osmotic pressure $\Delta \pi$ of the micelles. The lost membrane occupied volume $25 \mu \mathrm{m}^{2} \cdot 4 \mathrm{~nm}$. Subdividing into $N$ micelles of volume $\frac{4 \pi}{3} r_{m}{ }^{3}$ gives $N \approx 2 \cdot 10^{5}$. Supposing that half of these remain trapped in volume $\Delta V \approx 250 \mu \mathrm{m}^{3}$ while the other half escape, the volume fraction is then $4 \cdot 10^{-4}$. We may thus use the ideal gas formula (van 't Hoff's law) to get $\Delta \pi \approx k_{B} T N / 2 \Delta V=17 \mathrm{dyn} / \mathrm{cm}^{2}$.

In the presence of our hypothetical small micelles, the line tension is no longer a constant. The rapid jump to adhesion can trap micelles between the two vesicle walls. Eventually a micelle can incorporate into the outer wall, delivering its modified lipid contents and greatly decreasing the energy required to form an edge [16]. Thus we overcome the nucleation energy barrier, but only when an inner object sticks to the outer vesicle wall, as observed. As the perimeter of the pore increases, and as the impurities diffuse away from the edge, the effective $\gamma$ rises towards its nominal value $\gamma_{0}$. For a direct estimate of $\gamma_{0}$ in our experiment, we can examine the rapid completion stage of expulsion. Here the exit pore snaps shut, propelling the inner vesicle a few microns in one or two video frames. From Fig. 1 we estimate a speed $v \approx 3 \cdot 10^{-3} \mathrm{~cm} / \mathrm{s}$. Setting this equal to the Stokes drag on a sphere of radius $r=3.3 \mu \mathrm{m}$ gives the order-of-magnitude estimate for the line energy $\gamma_{0} \approx 10^{-7}$ dyn. Other events gave similar values.

Once the exit pore is established, we have argued that it should not seal tightly around the daughter vesicle but rather must leave a gap of width $w$. Indeed while the line energy $\gamma_{0}$ tries to close the gap, thermal fluctuations constantly keep it open. We can estimate

2 We thank J. Israelachvili, U. Seifert, and D. Zhelev for independently suggesting this mechanism to us. 
the average width $w$ of the gap by adapting Helfrich's "steric repulsion" argument [17] (see [18). At the halfway point of expulsion, the rim of the exit pore is a tense fluctuating line, and so it feels an effective repulsive free energy of $1.89 \cdot \frac{2 \pi r\left(k_{B} T\right)^{2}}{\gamma_{0} w^{2}}$ pushing it away from the daughter [18]. The line tension however creates a two-dimensional "disjoining pressure," providing another contribution to the effective free energy of $2 \pi \gamma_{0}(r+w)$. Minimizing the total free energy gives an average gap width of $w=60 \mathrm{~nm}$. A gap this wide certainly allows free and rapid diffusion of small solutes like sugar. Prior to the halfway point the pressure also helps close the gap; after the halfway point nothing keeps the gap closed so that expulsion finishes rapidly.

While $w$ turns out to be larger than twice our proposed micelle radius $2 r_{m} \approx 10 \mathrm{~nm}$, so that ultimately the micelles will simply escape, nevertheless initially there will be an osmotic pressure. Following the analysis of Finkelstein [19], let us first neglect the line tension altogether and consider the free flow at a leaky orifice with a concentration jump $\Delta c$. We take the orifice to be a slit of length $L=2 \pi r$ and width $w \ll L$. There will be no pressure gradient down the center of the slit, but the depletion zone of width $r_{m}$ will feel the van 't Hoff pressure jump $\Delta \pi \approx 17 \mathrm{dyn} / \mathrm{cm}^{2}$ estimated earlier, leading to a fluid velocity $v \approx \frac{\Delta \pi}{\eta} r_{m}$ at the edge of the depletion zone. Here $\eta=0.78 \mathrm{cP}$ is the viscosity of water at $31^{\circ} \mathrm{C}$. Thus our approximate prediction for the flow rate is $Q \approx$ $L w v \approx 14 \mu \mathrm{m}^{3} / \mathrm{s}$, comparable to though somewhat less than the observed initial inward flow of about $100 \mu \mathrm{m}^{3} / \mathrm{s}$ (Fig. 3b). Our estimate of $Q$ could be improved by accounting for hydrodynamic interactions, which effectively increase the width of the depletion zone, and thus increase the velocity and $Q$.

We neglected the line energy $\gamma_{0}$ in the above estimate. The inward flow $Q$ just estimated will be partly cancelled by an outward flow due to the interior pressure $p \approx$ $\gamma_{0} / r^{2} \approx 1 \mathrm{dyn} / \mathrm{cm}^{2}$, reflecting the force needed to open the pore. The two flows balance

when $p=\Delta \pi \frac{2 r_{m}}{w}$ 19. Using our estimate of $\Delta \pi$ we see that indeed the line tension is unable to stop the inward flow.

\section{Conclusion}

Spontaneous vesicle expulsion is a surprisingly complex behavior to emerge from such a simple system, consisting of just water plus lipid. We have seen how laser tweezers act on lipid bilayers to create a simple micron-scale machine, which pulls in material, repackages it, and pumps water against a pressure gradient. Such machines are of fundamental interest 
for the light they may shed on processes in living systems. Expulsion also hints at the exciting practical possibility of transforming membrane structure when and where we wish to do so. Further progress will require a better characterization of the objects created by the laser, perhaps by light scattering from the interior of the outer vesicle, and a more sophisticated theory, e.g. treating transport through a fluctuating gap.

\section{Acknowledgments}

We would like to thank R. Bruinsma, N. Dan, D. Fygenson, M. Goulian, R. Granek, S. Gruner, J. Israelachvili, R. Kamien, M. Kraus, N. Levit, A. Libchaber, R. Lipowsky, T. Lubensky, R. Menes, S. Milner, C. Peskin, S. Safran, U. Seifert, V. Suraiya, and D. Zhelev for their help. This work was supported in part by the Minerva Center for Nonlinear Science, the Minerva Foundation (München, Germany), the US/Israeli Binational Foundation grant 94-00190, and NSF grant DMR95-07366. EM was supported in part by a Madeleine Haas Russell Chair; JDM was supported in part by an FCAR Graduate Fellowship from the government of Quebec. 


\section{References}

[1] B. Alberts, D. Bray, J. Lewis, M. Raff, K. Roberts, and J. Watson, Molecular biology of the cell (Garland, 1989).

[2] E. Evans and W. Rawicz, "Entropy-driven tension and bending elasticity in condensedfluid membranes," Phys. Rev. Lett. 64 (1990) 2094.

[3] R. Bar-Ziv and E. Moses, "Instability and 'pearling' states produced in tubular membranes by competition of curvature and tension," Phys. Rev. Lett., 73 (1994) 1392.

[4] R. Bar-Ziv, R. Menes, E. Moses, and S. Safran, "Local unbinding of pinched membranes," Phys. Rev. Lett. 75 (1995) 3356.

[5] P. Nelson, T. Powers, and U. Seifert, "Dynamic theory of pearling instability in cylindrical vesicles," Phys. Rev. Lett. 74 (1995) 3384; R. Goldstein, P. Nelson, T. Powers, and U. Seifert, "Front propagation and the pearling instability of tubular vesicles," J. Physique II (France) 6 (1996) 767.

[6] R. Granek and Z. Olami, "Dynamics of Rayleigh-like instability induced by laser tweezers in tubular vesicles of self-assembled membranes," J. Phys. II France 5 (1995) 1348.

[7] R. Bar-Ziv, T. Frisch, and E. Moses, "Entropic expulsion in vesicles," Phys. Rev. Lett. 75 (1995) 3481.

[8] F. Menger and K. Gabrielson, "Chemically-induced birthing and foraging in vesicle systems," J. Am. Chem. Soc. 116 (1994) 1567.

[9] D. Fygenson, private communication.

[10] D. Needham and E. Evans, Biochemistry, 27 (1988) 8261; H. P. Duwe, J. Kas and E. Sackmann, J. Phys. (France) 51 (1990) 945; M. Mutz and W. Helfrich, J. Phys. (France) 51 (1990) 991.

[11] S.M. Block, in Noninvasive techniques in cell biology, 375-402, (Wiley-Liss, 1990); K. Svoboda and S. M. Block, Annu. Rev. Biophys. Biomol. Struct., 23 (1994) 247; A. Simon, Ph.D Thesis, Univ. of Chicago, (June, 1992).

[12] E. Evans, "Detailed mechanics of membrane-membrane adhesion and separation," Biophys. J. 48 (1985) 175; W. Helfrich, "Tension-induced mutual adhesion and a conjectured superstructure of lipid membranes," in Handbook of biological physics, ed. R. Lipowsky and E. Sackmann (Elsevier, 1995) pp. 691-721.

[13] C. Taupin, M. Dvolaitzky, and C. Sauterey, "Osmotic pressure induced pores in phospholipid vesicles," Biochemistry 14 (1975) 4771.

[14] D. Needham, in Permeability and stability of lipid bilayers, ed. E. Disalvo and S. Simon (CRC Press, 1995) p. 69.

[15] W. Harbich and W. Helfrich, "Alignment and opening of giant lecithin vesicles by electric fields," Z. Naturforsch. 34a (1979) 1062. 
[16] L. Chernomordik, G. Melikyan, and Y. Chizmadzhev, "Biomembrane fusion," Biochim. Bioph. Acta 906 (1987) 309.

[17] W. Helfrich, Z. Naturforsch. 33a (1978) 305.

[18] R. Lipowsky, "Generic interactions of flexible membranes," in Handbook of biological physics, ed. R. Lipowsky and E. Sackmann (Elsevier, 1995) pp. 548, 554.

[19] A. Finkelstein, Water movement through lipid bilayers, pores, and plasma membranes, (Wiley, 1987). 